\section{$1673 f$ NETWORK ON THE COORDINATION AND HARMONISATION OF EUROPEAN OCCUPATIONAL COHORTS (OMEGA-NET)}

IS Mehlum. National Institute of Occupational Health (STAMI), Oslo, Norway

10.1136/oemed-2018-ICOHabstracts.356

OMEGA-NET is a 4 year COST Action network, starting in October 2017, funded by EU. Occupation and paid employment is an essential component of adult life and a major determinant of health and healthy ageing. However, in recent years there has been very limited coordination and promotion of European health research on occupation and employment. Europe currently has some of the most valuable occupational, industrial, and population cohorts worldwide. The lack of integration of these cohorts hampers the optimal exploitation of these resources, essential to underpin evidence-based interventions and policy. The overarching concept of OMEGANET is to create a network to optimise the use of occupational, industrial, and population cohorts at the European level. OMEGA-NET will advance i) collaboration of existing cohorts, with extensive contemporary information on employment and occupational exposures, ii) coordination and harmonisation of occupational exposure assessment efforts, and iii) facilitation of an integrated research strategy for occupational health in Europe. We will inventory numerous cohorts with occupational information in Europe; implement an online interactive tool with detailed information on existing cohorts; facilitate work on harmonisation of occupational exposure and health outcome information and new protocols for data collection; connect scientific communities on occupational health in Europe and beyond; and provide networking, leadership, and training opportunities for early career researchers in occupational epidemiology and exposure assessment. Collaboration through OMEGA-NET will enhance the scientific output from individual studies and facilitate pooled studies, data sharing, and transfer of tools and skills to make greater and more efficient use of existing cohorts. The work will provide a foundation for an enhanced evidence base for the identification of health risks and gains related to occupation and employment to foster safe and healthy preventive strategies and policies. Researchers from countries outside Europe can participate in COST Actions on the basis of ascertained mutual benefit.

\section{CHALLENGES IN OCCUPATIONAL HEALTH JOURNAL PUBLISHING}

${ }^{1} \mathrm{MR}$ Sim, ${ }^{2} \mathrm{~T}$ Guidotti, ${ }^{3} \mathrm{M}$ Härmä, ${ }^{4} \mathrm{~J}$ Hobson, ${ }^{5} \mathrm{SK}$ Kang. 'Monash University, Melbourne, Australia; ${ }^{2}$ Occupational + Environmental Health and Medicine, Washington D.C., USA; ${ }^{3}$ Finnish Institute of Occupational Health, Helsinki, Finland; ${ }^{4}$ Hobson Health, Stoke on Trent, UK; ${ }^{5}$ Gachon University Gil Medical Centre, Incheon, Rep of Korea

\subsection{6/oemed-2018-ICOHabstracts.357}

Publishing in peer reviewed scientific journals has been the traditional method to provide the research findings needed to improve occupational health practice, develop workplace exposure limits and inform policy development to better control workplace hazards. In recent years, journal publishing has faced several challenges in maintaining their influence and independence in the occupational health field. A major challenge is managing actual and perceived conflicts of interest and striking the right balance between input by industry and government in occupational health research, while preserving researcher independence. In recent years here has been a growing number of open access journals which are funded by authors paying a processing charge instead of the traditional method whereby funding comes from reader subscriptions. While there are many legitimate open access journals, this trend has given rise to what are known as 'predatory journals', which are established with the main purpose of making a profit, often having little involvement within the occupational health community and little regard for rigorous peer review. This endangers the quality of scientific publication. Another challenge for journals is how to better engage with researchers and practitioners in low and middle income countries where higher workplace hazards are more likely to occur. Content sharing to allow greater access to peer reviewed journal articles is another challenge for journals, especially as it is becoming more common for research grant bodies to require wide dissemination of the findings from studies they fund, which can conflict with journal publishing and access policies. To help editors and journals deal with the wide range of problems which can arise in the publishing process, the Committee on Publication Ethics has been established, which provides useful case studies and other resources. These issues and more will be the main focus of this workshop, with contributions by Editors from some of the main international occupational health journals.

\section{COCHRANE WORK}

Deirdre Fitz Gerald. Medmark Occupational Healthcare Cork Ireland

\subsection{6/oemed-2018-ICOHabstracts.358}

Aim of special session To introduce and promote Cochrane Work, including issues relating to the development of and quality requirements of systematic reviews.

${ }^{1} \mathrm{JH}$ Ruotsalainen, ${ }^{2} \mathrm{HF}$ van der Molen, ${ }^{3} \mathrm{TC}$ Morata, ${ }^{4}$ Prof Carel $\mathrm{T}$ Hulshof, ${ }^{5} \mathrm{R}$ Sauni, ${ }^{6}$ Deirdre FitzGerald

${ }^{1}$ Cochrane Work, Finnish Institute of Occupational Health, Kuopio, Finland

${ }^{2}$ Academic Medical Centre, University of Amsterdam, Department, Coronel Institute of Occupational Health, Amsterdam Public Health research institute, Amsterdam, The Netherlands

${ }^{3}$ National Institute for Occupational Safety and Health, Cincinnati, USA

${ }^{4}$ Netherlands Society of Occupational Medicine (NVAB), Centre of Excellence, Utrecht, the Netherlands; Academic Medical Centre, dept. Coronel Institute of Occupational Health, Amsterdam, The Netherlands

${ }^{5}$ Department for Occupational Safety and Health, Ministry of Social Affairs and Health, Finland

${ }^{6}$ Medmark Occupational Healthcare, Cork, Ireland

\section{0a THE PICO QUESTION IS TO A COCHRANE REVIEW LIKE THE THICK CREAMY HEAD IS TO A PINT OF GUINNESS}

JH Ruotsalainen. Cochrane Work, Finnish Institute of Occupational Health, Kuopio, Finlan

\subsection{6/oemed-2018-ICOHabstracts.359}

Introduction Conducting Cochrane reviews and updating them regularly requires a considerable investment from authors and 